\title{
Some considerations for different time-domain signal processing of pulse compression radar
}

\author{
Miguel A. Cabrera ${ }^{1,2}$, Enrico Zuccheretti ${ }^{3, \star}$, Rodolfo G. Ezquer ${ }^{1,2,4}$, Umberto Sciacca ${ }^{3}$, Joachin M. Lopez ${ }^{2}$, \\ Maria G. Molina ${ }^{1}$, James A. Baskaradas ${ }^{3}$ \\ ${ }^{1}$ Laboratorio de Ionósfera, Dpto. de Física, FACET, Universidad Nacional de Tucumán, Tucumán, Argentina \\ ${ }^{2}$ Centro de Investigación sobre Atmósfera Superior y Radiopropagación, Universidad Tecnológica Nacional, Tucumán, Argentina \\ ${ }^{3}$ Istituto Nazionale di Geofisica e Vulcanologia, Rome, Italy \\ ${ }^{4}$ Consejo Nacional de Investigaciones Cientificas y Técnicas (CONICET), Buenos Aires, Argentina
}

\section{Article history}

Received May 28, 2010; accepted September 15, 2010.

Subject classification:

Complementary code, Correlation, Ionosphere, Radar, Time domain.

\section{ABSTRACT}

Radar technology has for a long time used various systems that allow detection under high-resolution conditions, while emitting at the same time low peak power. Among these systems, transmitted pulse encoding by means of biphasic codes has been used for the advanced ionospheric sounder that was developed by the AIS-INGV ionosonde. In the receiving process, suitable decoding of the signal must be accomplished. This can be achieved in both the time and the frequency domains. Focusing on the time domain, different approaches are possible. In this study, two of these approaches have been compared, using data acquired by the AIS-INGV and processed by means of software tools (mainly Mathcad $\left.{ }^{(}\right)$. The analysis reveals the differences under both noiseless and noisy conditions, although this does not allow the conclusive establishment as to which method is better, as each of them has benefits and drawbacks.

\section{Introduction}

The envelope detection technique has been the traditional method used as ionospheric sounders in analog radar applications. Nowadays, there are new solutions that are based on the spread-spectrum technique and that allow high-range resolution to be reached with low emitted power. Among these, phase modulation with a biphasic code allows a bandwidth to be obtained that is similar to that of narrowpulse envelope detection.

The advanced ionospheric sounder developed by the Istituto Nazionale di Geofisica e Vulcanologia (AIS-INGV) is based on this kind of modulation. Its receiver exploits a triple conversion technique, to obtain a final intermediate frequency (IF) at $100 \mathrm{kHz}$. The relevant information is then acquired directly at this level by I-Q sampling [Zuccheretti et al. 2003]. Standard ionospheric sounding is performed by a frequency sweep along the high frequency (HF) band; specific experiments can be carried out at fixed frequencies.

This study deals with two signal-detection processes in a discrete time domain based on measurements obtained with the AIS-INGV placed in the ionospheric observatory in Rome. Different conditions have been examined, with and without environmental noise. The noise conditions are those typical of normal soundings. We examined signals with low and high noise levels. The noiseless condition was accomplished in the so-called "closed-loop» configuration, i.e. without emitting radiofrequency (RF) power into the ionosphere, but by connecting the transmitter output directly to the receiver (suitably attenuated).

To extract the relevant information from the echo signals, processing methods are needed. Algorithms were implemented using the Mathcad ${ }^{\complement}$ software. The simulation allows an analysis of the signals involved along the process, to establish qualitative and quantitative criteria of the signal.

\section{Signal coding for radar applications}

From the beginning of ionospheric research, high frequency radars, known as ionospheric sounders, have been based on pulse repetition and envelope-detection techniques. To make weak-echo detection possible, high-power radio signals need to be radiated. Modern radars for geophysical applications use the spread-spectrum technique to improve the range resolution, by transmitting low power [Barry 1971, Poole 1985, Bianchi and Altadill 2005, among others].

While emitting low peak power, it is possible to optimize the range resolution and the signal-to-noise ratio (SNR) using correctly encoded long radar pulses. Signal coding methods are aimed at obtaining a bandwidth that is as wide as in the envelope-detection technique, with a narrow pulse. This technique is known as "pulse compression» and it 

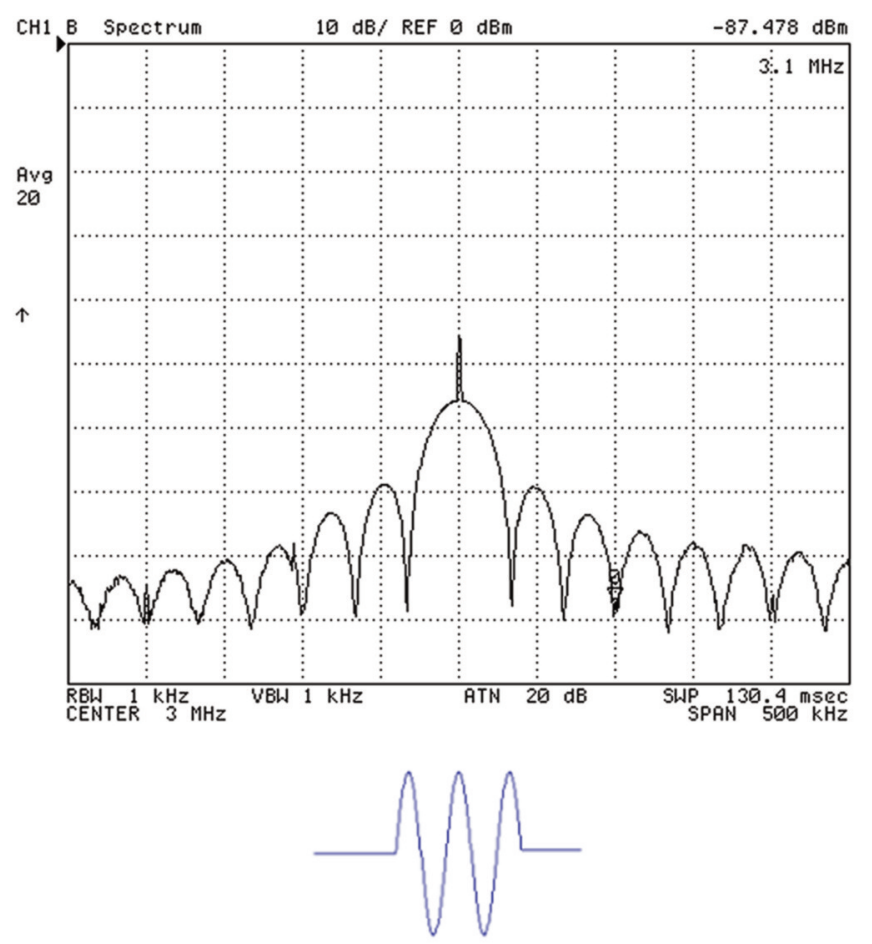

a)

Figure 1. Spectra and time shapes of a carrier under different coding conditions (with the same bandwidth). (a) Not coded (pulse width: $30 \mu \mathrm{s}$ ). (b) Binary encoded with complementary code (pulse width: $480 \mu$ s).

makes it possible to obtain high-range resolution with long transmitted pulses. It is based on the generation of longduration waveforms, transmission of encoded carriers, and processing of the signals received through mathematical algorithms, and particularly correlation [Rastogi 1990, Mohamed 1991, among others]. The range resolution is related to the minimum duration of a code element, usually called the «subpulse» [Patro et al. 1990].

Figure 1 shows experimental spectrum analyzer observations for a short high-power-burst carrier (Figure 1a) and a binary encoded long one (Figure $1 \mathrm{~b}$ ). The qualitative waveforms of the pulses are shown in the lower part of Figure 1. There are equivalent effects from the spectral point of view.

The data analyzed in this study were obtained by means of the AIS-INGV ionosonde, where 16-bit complementary codes were used. They have a $30-\mu$ s subpulse length, and a 4.5-km range [Baskaradas et al. 2002, Zuccheretti et al. 2003].

\section{Echo detection}

When using binary encoded carriers, signal processing methods are necessary to extract information from the encoded signals received, and to obtain the echo delay time and the energy amplitude [Bianchi et al. 2003, among others]. To develop such a system, considerations of the theoretical and practical aspects of radio signals management are needed, to detect weak and noisy signals, like those used in radar design [Skolnik 1980, Skolnik 1990, Curry 2005]. As a part of the detection process, correlation is the mathematic tool that allows information to be extracting from the echo.
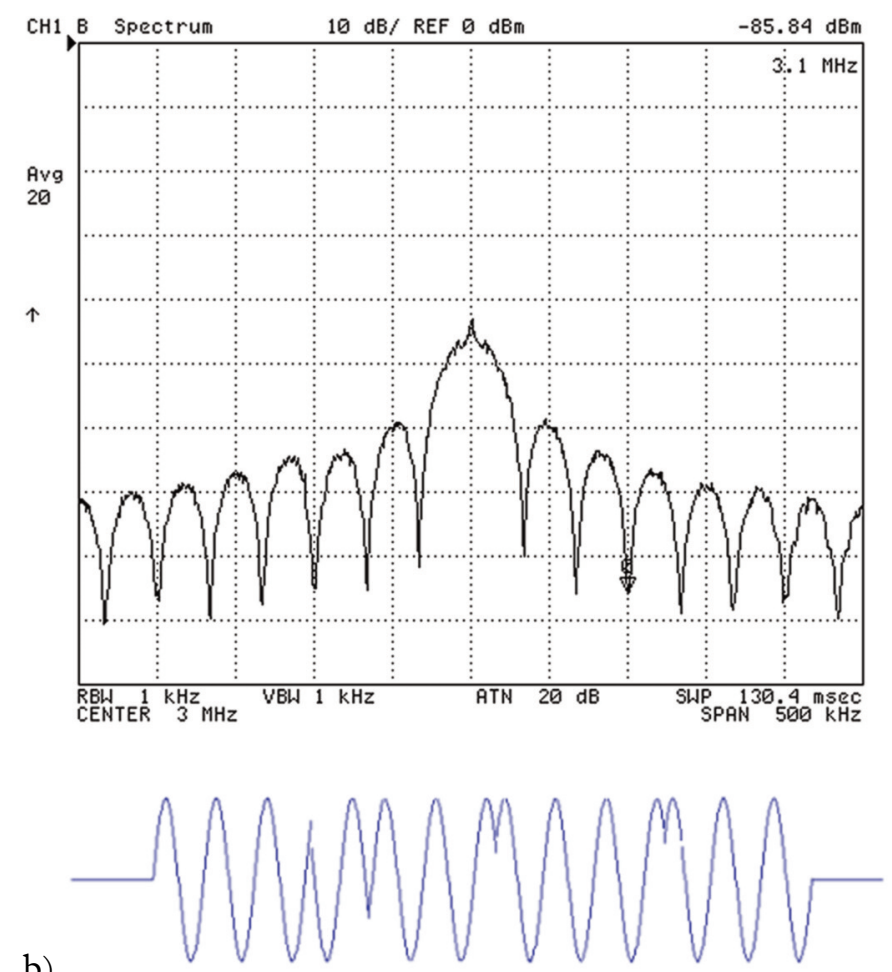

Thus, after filtering, amplifying and down-converting, quadrature sampling is used to retrieve the information from the IF [Tomasi 1996], in I and Q digital samples. Correlating the output with the code the echo peak is obtained.

Two methods are used to detect the echo peak. The first is shown in Figure 2; this combines the $I$ and $Q$ channels to obtain the complex amplitude, then the correlation with the code is performed [Skolnik 1980]. To keep the code information, correct sign recovery is necessary before performing the correlation. For this, the sign is taken from one channel (e.g. channel «I»). Hereafter, this method will be called module-based correlation (MBC).

The second method is shown in Figure 3, where the I and $Q$ channels are correlated with the code separately, and the amplitude is obtained at the end of the process [Skolnik 1990]. Hereafter, this method will be called quadrature component correlation (QCC). In Figures 2 and 3, the asterisk (*) denotes the point where the mathematical analysis starts.

It is well known that there are several codes that are used in radar applications [Ioannidis and Farley 1972]. In the present study, the complementary code sequence is used. Complementary code pairs have the important property that the sum of the auto-correlation functions of each sequence is equal to zero for all lags, except for a zero lag [Golay 1961]. General criteria in high-atmosphere radar design suggest the use of codes with autocorrelation functions with a high principal lobe and minimum side lobes, to obtain defined echoes from noisy received signals. Sultzer and Woodman [1984] specified that the total power in the correlation side lobes should be $\leq 20 \%$ of the main lobe. Thus, comparing 


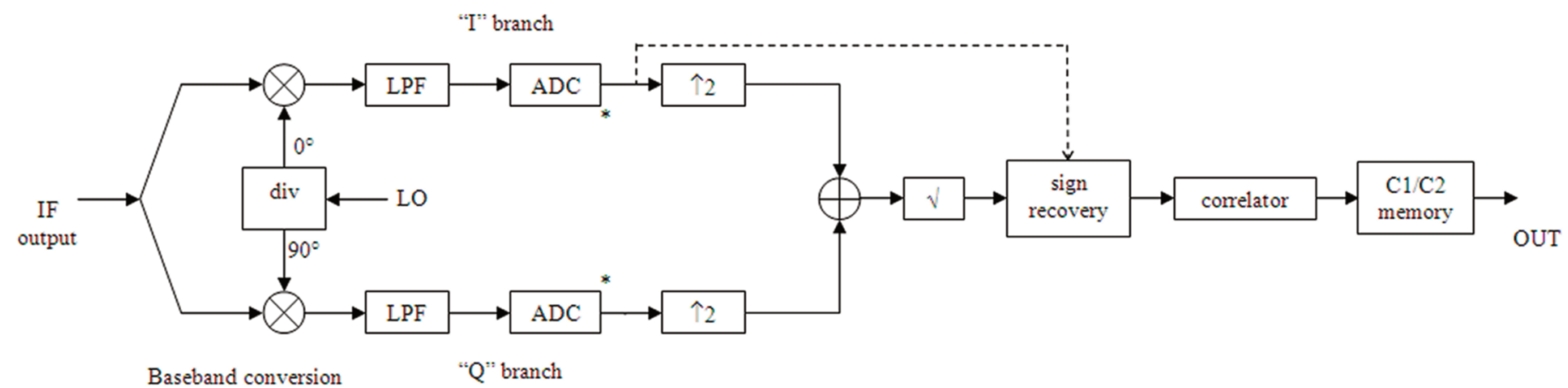

Figure 2. Schematic diagram of the MBC method. *, point where mathematical analysis starts; div, power divider; LO, local oscillator; LPF, low pass filter; ADC, analog-to-digital converter.

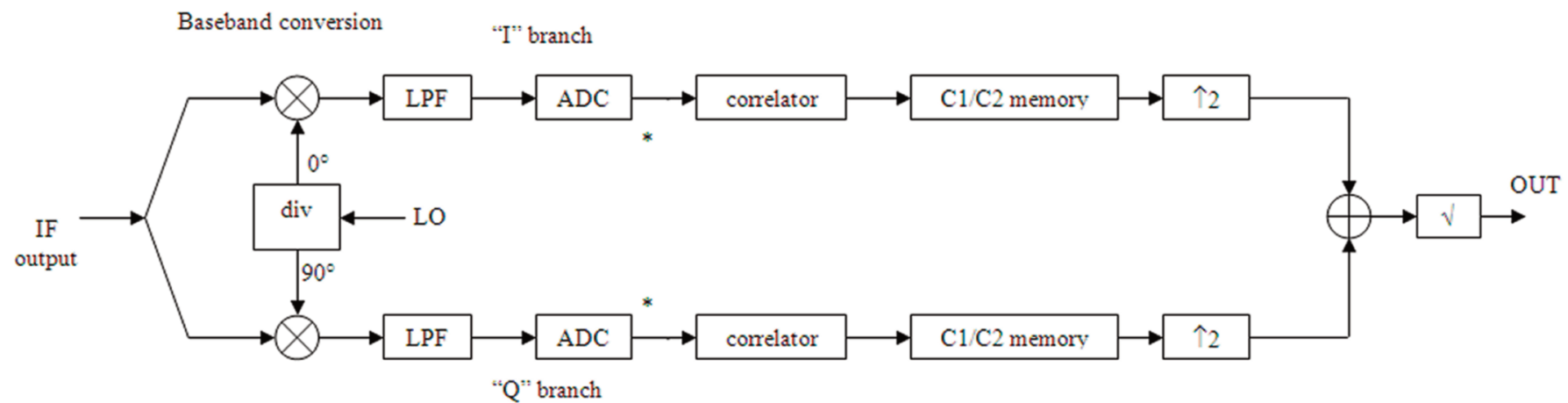

Figure 3. Schematic diagram of the QCC method. *, point where mathematical analysis starts; div, power divider; LO, local oscillator; LPF, low pass filter; ADC, analog-to-digital converter.

autocorrelation functions of different sequences provides useful information when choosing suitable binary sequences for radar systems.

Simulation is a tool to validate hypotheses before system implementation. The model provides a comprehensive way to understand the process, and to analyze the relationships between the stages and parameters in the design that could be developed into an actual system. The software tools have central roles in the modeling process. They can build results that can be input into the model (like feedback), to create new results to adjust and validate. In the further step, it will be possible to develop the actual system.

Mathcad ${ }^{\odot}$ has been used to model some of the stages and signals involved in processes, such as code reconstruction with quadrature sampling, and correlation and integration processes. It is well known that, by using frequency downconversion process, it is possible to shift signals from radio frequency $(\mathrm{RF})$ values to a fixed IF value, maintaining the same band. So, the bandwidth and the original information in the carrier signal is kept. In the present study, the code reconstruction is performed at the IF of $f=100 \mathrm{kHz}$ [Baskaradas et al. 2002].

\section{Overview of the signals}

In this section, the signals involved are presented in a theoretical way, to show the process and to obtain the echo delay time and the amplitude information. In general, it is possible to consider a carrier signal $a(t)$ in the continuous time domain as follows:

$$
a(t)=A e^{j \omega_{0} t},
$$

where $A$ is the amplitude, $j=\sqrt{-1}$ is the imaginary unit, $f_{0}$ is the frequency $(\mathrm{Hz})$ and $\omega_{0}=2 \pi f_{0}$ the angular frequency. When $a(t)$ is modulated with the code $C(t)$, we can write the transmitted signal $s(t)$ as follows:

$$
s(t)=A \cdot C(t) e^{j \omega_{0} t} .
$$

After the ionospheric reflections, the received signal $r(t)$ will be the sum of multiple attenuated and shifted echoes. It is possible to write this as:

$$
r(t)=\sum_{i=1}^{n} b_{i} s\left(t-\tau_{i}\right)
$$

where $b_{i}$ and $\tau_{i}$ are the $i$-th order values of the attenuation factor and time delay due to total reflected signal contributions. For this simulation we adopted $n=1$ (unique way), and thus Equation (4.1) becomes:

$$
r(t)=b_{1} \cdot A \cdot C\left(t-\tau_{1}\right) e^{j \omega_{0}\left(t-\tau_{1}\right)} .
$$

The quadrature sampling removes the carrier, and after this process, the phase component in the signal will be 
represented by the complex amplitude coefficient $\beta$. Now it is possible to redefine the signal $r(t)$ in the continuous time domain by means of $r[n]$ in the discrete time domain, as follows:

$$
r[n]=\beta C\left[n-d_{1}\right] .
$$

To extract the delay $d_{1}$ from this equation, a discrete time-domain correlation process with local modulator code samples $C[n]$ is performed, defined as:

$$
r_{c}[n]=r[n] * C[n] .
$$

Performing this operation over the received sampled signal with the code, the following expression is obtained:

$$
r_{c}[n]=K \beta \delta\left(n-d_{1}\right),
$$

where $d_{1}$ represents the only delay considered, the value of the impulsive function $\delta\left(n-d_{1}\right)$ is 1 or 0 , depending on the lag in the correlation function, and $K$ is the number of bits that constitute the code. Using our complementary phase code, if noise is not present, the side lobes are eliminated in principle, so Equation (4.2) represents the exact result.

$K$ represents a gain for the recovered signal, although when the noise is present it is also increased, but with a gain of $\sqrt{K}$. This means a SNR gain [Ghebrebrhan et al. 2004]. In $\mathrm{dB}$, the power gain will be $(10 \log K+3)[\mathrm{dB}]$, where plus $3 \mathrm{~dB}$ is due to the benefit-adding sequence of the complementary codes [Bianchi et al. 2003].

For the sake of simplicity, in the mathematical expressions given above, the noise was avoided; however, the noise will be considered in the processing of the actual AISINGV echo in the following sections of this study.

\section{The MBC and QCC methods: amplitude considerations}

In the AIS-INGV system, two 16-bit complementary code sequences are generated, and a RF carrier is modulated by a biphasic shift key [Tomasi 1996] during two «transmitting windows» (or couple of shots) of $480 \mu$ s each. The transmitted signal is partially down-reflected by the ionospheric plasma, and a weak signal is received by the system. After the convenient filtering and down conversions, the carrier is removed and the quadrature sampling process is performed to restore the code. The sampled results are two signals: I and Q. In the following, the application of the MBC and QCC methods is described in more detail, to obtain the echo time delay $\tau$ and the amplitude. Note that the exact representation of the signal should be the one in Equation (4.2); «\$) and «Q» represent a concise way to denote the overall time behavior of the signals, and these will be used often to predict the amplitude of the peak of the output only, although it should not be forgotten that they are real signals: $I(t)$ and $Q(t)$.

In the MBC method, the $A_{1}$ and $A_{2}$ amplitudes for each sequence are obtained as follows:

$$
\begin{aligned}
& A_{1}=\sqrt{\left(I_{1}\right)^{2}+\left(Q_{1}\right)^{2}} \\
& A_{2}=\sqrt{\left(I_{2}\right)^{2}+\left(Q_{2}\right)^{2}}
\end{aligned}
$$

Then they are correlated with the corresponding code sequence as follows:

$$
\begin{aligned}
& \operatorname{Corr}_{1}=A_{1} * \operatorname{Cod}_{1}=K A_{1} \\
& \operatorname{Corr}_{2}=A_{2} * \operatorname{Cod}_{2}=K A_{2}
\end{aligned}
$$

Where $K$ is the correlation gain.

The total amplitude to obtain the benefits of the complementary code will be (from Equations 5.1 and 5.2):

$$
\begin{aligned}
& \text { MBCamp }=\text { Corr }_{1}+\text { Corr }_{2}= \\
& =K\left(A_{1}+A_{2}\right)=K\left(\sqrt{I_{1}^{2}+Q_{1}^{2}}+\sqrt{I_{2}^{2}+Q_{2}^{2}}\right)
\end{aligned}
$$

In the QCC method, the $I_{1}$ and $Q_{1}$ samples are correlated with $\operatorname{Cod}_{1}$ and $I_{2}$, and the $Q_{2}$ samples are correlated with $\operatorname{Cod}_{2}$. Then the signals procession is as follows:

$$
\begin{aligned}
& \operatorname{Corr}_{1}=I_{1} * \operatorname{Cod}_{1}=K I_{1} \\
& \operatorname{Corr}_{1}=Q_{1} * \operatorname{Cod}_{1}=K Q_{1} \\
& \operatorname{Corr}_{2}=I_{2} * \operatorname{Cod}_{2}=K I_{2} \\
& \operatorname{Corr}_{2}=Q_{2} * \operatorname{Cod}_{2}=K Q_{2}
\end{aligned}
$$

After correlation, the process continues in this way:

$$
\begin{aligned}
& \text { SumI }=\operatorname{CorrI}_{1}+\operatorname{CorrI}_{2}=K\left(I_{1}+I_{2}\right) \\
& \text { Sum } Q=\operatorname{Corr}_{1}+\operatorname{Corr}_{2}=K\left(Q_{1}+Q_{2}\right)
\end{aligned}
$$

Thus the complementary code benefits are obtained in this step. The results SumI and SumQ are obtained by algebraic summation because the $I_{1}$ and $I_{2}$ samples have the same phase, with the identical situation for the $Q_{1}$ and $Q_{2}$ samples. The next step is to combine the SumI and SumQ channelcorrelation results, to extract the amplitude, as follows:

$$
\text { QCCamp }=\sqrt{(\operatorname{SumI})^{2}+(\operatorname{Sum} Q)^{2}}
$$

This operation is possible because the correlation does not shift the original phase information in the $I$ and $Q$ channels, keeping the quadrature sampling properties.

As the correlation is a linear operation, it is possible to perform the algebraic summation of the in-phase samples as shown by Equation (5.5). To obtain the benefits of the complementary code, the complex summation will be done as in (5.6), which can be made more explicit as:

$$
\begin{aligned}
& \text { QCCamp }=K \sqrt{K^{2}\left(I_{1}+I_{2}\right)^{2}+K^{2}\left(Q_{1}+Q_{2}\right)^{2}}= \\
& =K \sqrt{I_{1}^{2}+I_{2}^{2}+2 I_{1} I_{2}+Q_{1}^{2}+Q_{2}^{2}+2 Q_{1} Q_{2}}
\end{aligned}
$$

It is possible to see that MBCamp and QCCamp are not 
equal. A more detailed analysis would show that the QCC amplitude is always less than that of the MBC (see Appendix A).

In general $I_{1} \neq I_{2}$ and $Q_{1} \neq Q_{2}$. Usually the target is fixed between two shots (i.e. code1 and code2), so it is possible to put $I_{1}=I_{2}$ and $Q_{1}=Q_{2}$; in this case Equations (5.3) and (5.7) become:

$$
\begin{gathered}
\text { MBCamp }=K\left(\sqrt{I_{1}^{2}+Q_{1}^{2}}+\sqrt{I_{2}^{2}+Q_{2}^{2}}\right)= \\
=K\left(2 \sqrt{I_{1}^{2}+Q_{1}^{2}}\right)=2 K A \\
\text { QCCamp }=K \sqrt{\left(I_{1}^{2}+I_{1}^{2}+2 I_{1} I_{1}+Q_{1}^{2}+Q_{1}^{2}+2 Q_{1} Q_{1}\right)} \\
=K\left(2 \sqrt{I_{1}^{2}+Q_{1}^{2}}\right)=2 K A
\end{gathered}
$$

So, in this ideal situation, the MBC and QCC methods show the same amplitude results. This condition with fixed targets is reached in a noiseless environment.

\section{Noise considerations}

Now an evaluation of the behavior with respect to noise will be carried out. Here, various simplifying hypotheses can be introduced, and as a consequence, the result is not applicable in the general situation, so it only gives an idea of the performance of the two systems:

1) The first hypothesis relating to the noise is that it is present without the signal. In this way, it is much simpler to evaluate the outputs. Given that the systems are not linear, the contemporary presence of the signal would give rise to mixed components in the output, which are difficult to evaluate.

2) The second hypothesis relates to the noise properties, whereby it is assumed that it enters the system in a point between the transmitter and the receiver, that it is Gaussian with a zero mean value, and that it is "white noise», i.e. it has a power spectrum density that is constant with respect to the frequency. Ideally, the bandwidth of this kind of noise is infinite, although for the purposes of our calculations here, it is sufficient that it is constant over all of the bandwidth of the filters (the IF narrow-band filter or the correlator); what happens outside of this has no influence. If the noise has a non-zero mean value, it can be set to zero at the points marked in Figures 2 and 3 with an asterisk $\left(^{*}\right)$.

3) It is assumed that the correlator filter has a flat response over its entire pass band. This is approximately true; however, a more precise calculation can be carried on simply considering the so called 'equivalent noise bandwidth', and the results would be substantially the same.

4) The noise exiting from the two mixers on the $I$ and $Q$ branches can be considered to be either uncorrelated or not; both hypotheses will be considered.

5) The sign recovery in the MBC is assumed to be ideal, i.e. it does not introduce additional noise.

It is possible to analyze what happens when noise passes through the various types of fundamental blocks appearing in the block diagrams, and then to predict the output noise power of the two systems. These calculations are performed in Appendix B.

The result is that the output noise variances are the same. Considering the mean value, the two systems do not produce the same result. Anyway, this "output" value provides no information about the signal, and it can be subtracted easily (even though it can be used to infer the noise power at the input).

In conclusion, the two systems appear to show similar behaviors with respect to the noise, assuming that all of the initial hypotheses are valid; they do appear to be reasonably valid. The only assumption that has been forced to be true is that the sign reconstruction in the MBC is perfect. Actually, the noise existing at the point used to evaluate the sign makes the reconstruction uncertain; this appears in the output as increased noise, so the MBC method is less reliable under noisy conditions.

\section{Some results}

In this section, the results are presented for a closedloop circuit in the instrument and for actual echo samples with low and high noise levels in the signals received. The measurements are performed by soundings at a fixed frequency of $3.5 \mathrm{MHz}$ for about $5 \mathrm{~s}$. In the accompanying Figures, the results of the time domain processes are shown. The horizontal positions of the correlation peaks indicate the heights of the target (actual ionospheric layer, as well as simulated echo), while the energy received is related to the peak amplitude, i.e. the distance between the absolute maximum and the average value. Furthermore, for both of these methods, the SNRs have been evaluated as $\mathrm{SNR}=10 \log A^{2} / \sigma^{2}$, with $\sigma^{2}$ as the variance of the timedomain series that represents the correlation. The variance is calculated as reported in Appendix C.

Figure 4 shows the results for the MBC and QCC methods in the discrete time domain for the AIS-INGV generated closedloop signals, with a programmed time delay. These results for the two methods are presented under condition of no integrations. The peak position corresponds to a programmed virtual height of about $204 \mathrm{~km}$. The differences in the amplitude values show some gain for the MBC method with respect to the QCC method: $(\mathrm{MBC}-\mathrm{QCC}) / \mathrm{QCC}=4.7 \%$. Therefore, under ideal noiseless conditions, the SNR values are in practice the same.

In Figure 5, the MBC algorithm shows higher gain with respect to the QCC method. Nevertheless, the differences in SNR values reveals the advantage of the QCC method compared to the MBC method.

Under highly noisy conditions, pulse integration has to be applied to clean the signals and to make the echo detection more reliable. In the present study, 10, 20 and 60 integrations were used (Figures 6 and 7). Using this process, 

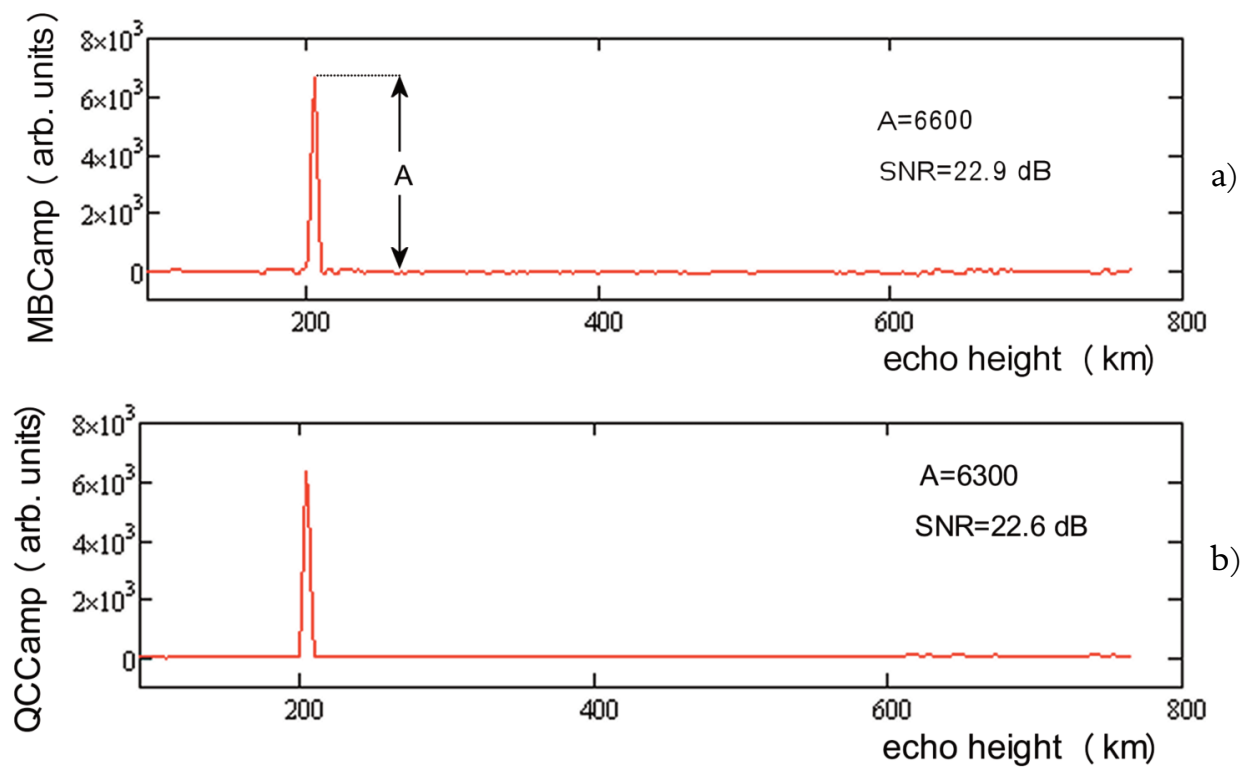

Figure 4. Discrete time domain processing on an AIS-INGV generated closed-loop signal. (a) MBC method. (b) QCC method.
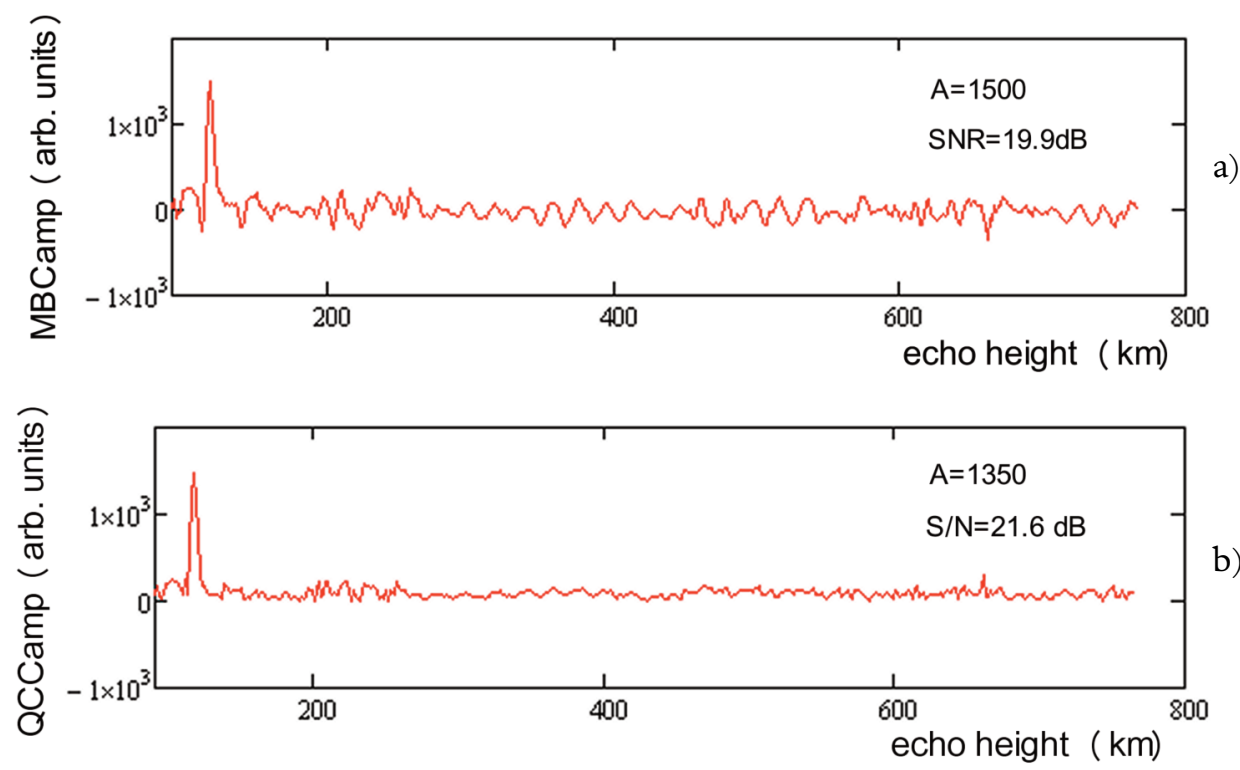

Figure 5. Results for actual AIS-INGV low-noise echo signals. (a) MBC algorithm. (b) QCC algorithm.

it is possible to obtain an extra gain of $20 \log \sqrt{Z}$, where $Z$ is the number of integrations [Skolnik 1990]. Under this condition, the differences between the SNR values for the MBC and QCC methods show that the latter works better.

\section{Conclusions}

Some of the steps of the processing operation procedure of the echo signal of a digital ionospheric sounder were modeled with Mathcad ${ }^{\odot}$ software, including: correlation processes, amplitude reconstructions and echo integration.

The preliminary results obtained in this study for the considered AIS-INGV echo signals show that it is possible to extract amplitude and delay-time information from noisy signals using discrete time-domain signal processing techniques. Among the different methods to accomplish this, two algorithms in the time domain were considered. These showed different behaviours, according to the operative conditions.

Under noiseless conditions, the methods are equivalent, and they assume a perfectly stable echo, i.e. the target remains fixed during the time interval between the transmission of the two codes. In contrast, when the phase difference between received codes 1 and 2 increases, the MBC method shows slight better behavior, as analyzed in Appendix A.

The presence of noise makes the sign recovery in the $\mathrm{MBC}$ algorithm troublesome, with the consequence that there is a worsening of the SNR. The better behavior of the QCC method is more evident under high noise conditions, so a smaller number of integrations is necessary to allow the signal to emerge from the noise. So, under actual 


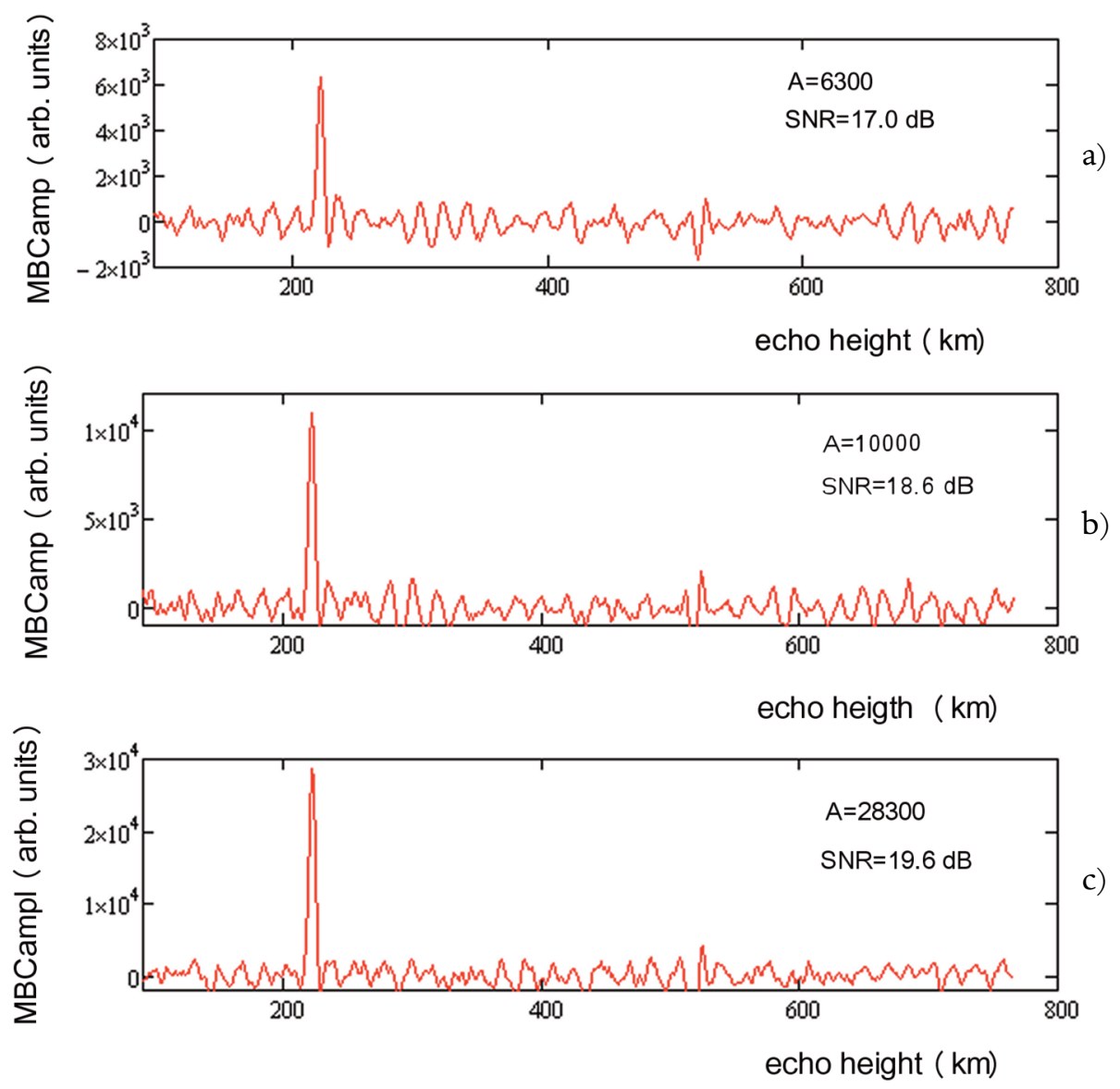

Figure 6. MBC results for an actual noisy echo signal, for 10 (a), 20 (b) and 60 (c) integrated received pulses.
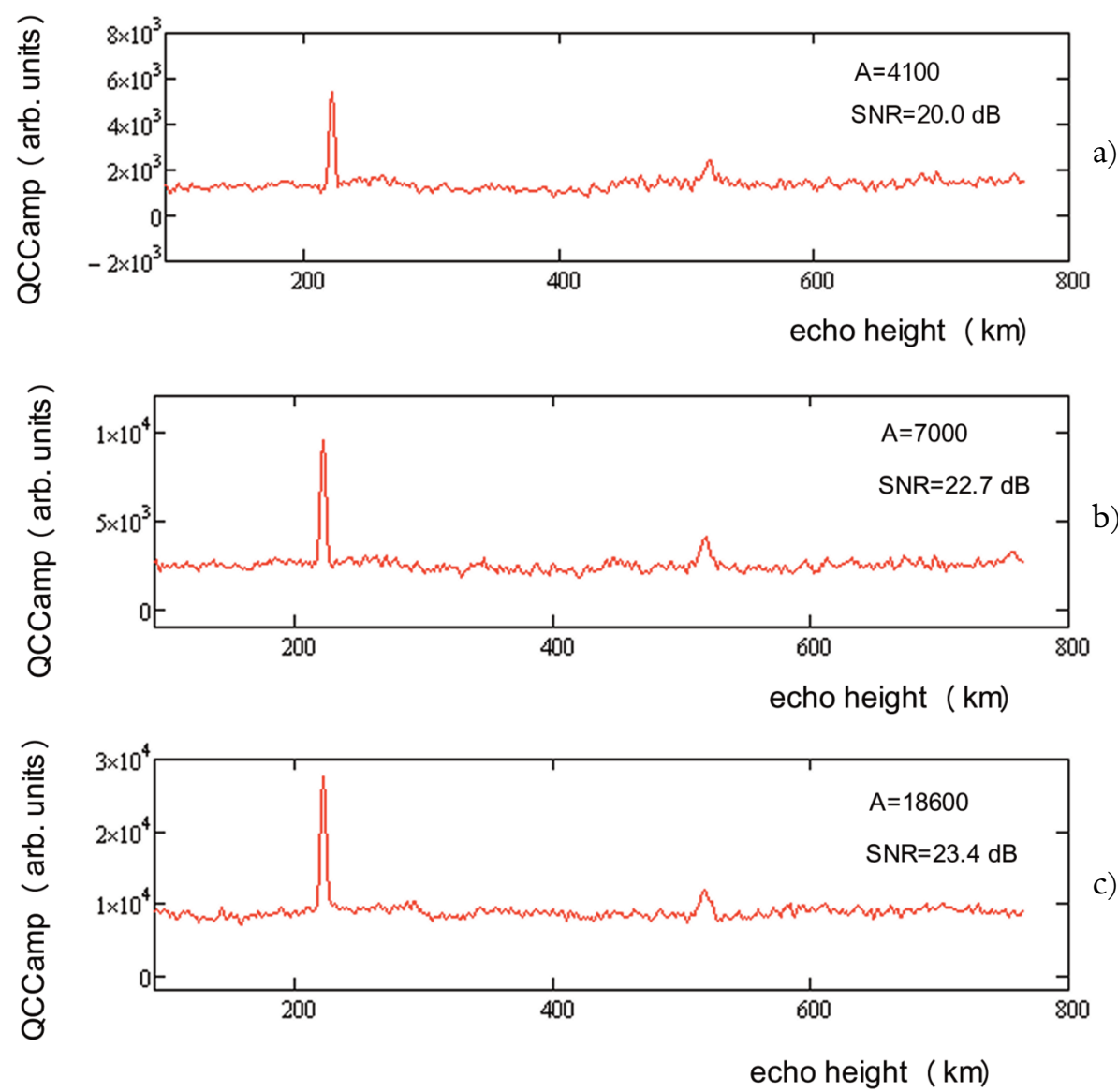

Figure 7. QCC results for an actual noisy echo signal, for 10 (a), 20 (b) and 60 (c) integrated received pulses. 
conditions, the QCC method is preferable, even though it requires more calculations.

The data presented in this study are not sufficient to definitely establish which of the MBC and QCC methods is better. In particular, the contemporary presence of noise and phase shifts between codes should be better investigated. Another situation that can be investigated is the presence of multiple echoes in the received signal.

Acknowledgements. This study was partially supported by projects 26/408 Universidad Nacional de Tucumán (Arg.), 25/P028 Universidad Tecnológica Nacional (Arg.) and the Istituto Nazionale di Geofisica e Vulcanologia, Rome, Italy.

\section{References}

Barry, G.H. (1971). A low power vertical incidence ionosonde, IEEE T. Geosci. Elect., 9 (2), 86-89.

Baskaradas Arokiasamy, J., C. Bianchi, U. Sciacca, G. Tutone and E. Zuccheretti (2002). The new INGV digital ionosonde - design report, Rapporti Tecnici INGV, 12, 59 pp.

Bianchi, C., U. Sciacca, A. Zirizzotti, E. Zuccheretti and J.A. Baskaradas (2003). Signal processing techniques for phase-coded HF-VHF radars, Annals of Geophysics, 46 (4), 697-705.

Bianchi, C. and D. Altaldill (2005). Ionospheric Doppler measurements by means of HF-radar techniques, Annals of Geophysics, 48 (6), 989-993.

Carlson, A.B. (1986). Communication Systems: an Introduction to Signals and Noise in Electrical Communication, 3rd edition, New York, 686 pp.

Curry, G.R. (2005). Radar System Performance Modeling, 2nd edition, Norwood, MA, 400 pp.

Ghebrebrhan, O., H. Luce, M. Yamamoto and S. Fukao (2004). Interference suppression factor characteristics of complementary codes for ST / MST radar applications, Radio Sci., 39, RS3013; doi: 10.1029/2003RS002901.

Golay, M.J.E. (1961). Complementary series, IRE T. Inform. Theor., 7, 82-87.

Ioannidis, G. and D.T. Farley (1972). Incoherent scatter observations at Arecibo using compressed pulses, Radio Sci., 7, 763-766.

Mohamed, N.J. (1991). Resolution function of nonsinusoidal radar signals. II - Range-velocity resolution with pulse compression techniques, IEEE T. Electromagn. C., 33, 51-58.

Patro, Y.K.G., K.R. Suresh Nair and P. Balamuralidhar (1990). Effect of signal-path distortions on the decoding performance in a pulse compression radar system, Radio Sci., 25, 1095-1100.

Poole, A.W.V. (1985). Advanced sounding: the FMCW alternative, Radio Sci., 20, 1609-1616.

Rastogi, P.K. (1990). Signal processing and data analysis in middle atmosphere radar, Radio Sci., 25, 1071-1086.

Skolnik, M.I. (1980). Introduction to Radar Systems, 2nd edition, New York, 581 pp.
Skolnik, M.I. (1990). Radar Handbook, 2nd edition, New York. Sultzer, M.P. and R.F. Woodman (1984). Quasi-complementary codes: a new technique for radar sounding, Radio Sci., 19, 337-344.

Tomasi, W. (1996). Sistemas de Comunicaciones Electrónicas, Ed. Prentice Hall, Mexico, 858 pp.

Zingales, G. (1992). Misure Elettriche: Metodi e Strumenti, Torino, $504 \mathrm{pp}$.

Zuccheretti, E., G. Tutone, U. Sciacca, C. Bianchi, J.A. Baskaradas (2003). The new AIS-INGV digital ionosonde, Annals of Geophysics, 46 (4), 647-659.

\footnotetext{
${ }^{\star}$ Corresponding author: Enrico Zuccheretti, Istituto Nazionale di Geofisica e Vulcanologia, Rome, Italy; e-mail: enrico.zuccheretti@ingv.it.

C) 2010 by the Istituto Nazionale di Geofisica e Vulcanologia. All rights reserved.
} 


\section{Appendix A}

For a better understanding of the behavior of these two methods with respect to the peak amplitude of the output signal, some calculations are carried out in this Appendix. It is true that between the first and the second shots (when codes 1 and 2 are used), the reflected signals can be different. Despite this, it is very unlikely that the modifications affect the amplitude of the received signal; it is more likely that they will modify the phase (Figure 8).

Each vector has a module and a phase. Assuming that the phase in the transmission pulse can jump between $0^{\circ}$ and $180^{\circ}$, after reflecting on the ionosphere it can vary from $\varphi$ to $\varphi+180^{\circ}$. The amplitude can also vary (the power received is obviously much less than that transmitted). When the receiving system extracts the $I$ and $Q$ components of the signal, these are simply:

$$
\begin{aligned}
I(t) & =A(t) \cos \varphi(t) \\
Q(t) & =A(t) \sin \varphi(t)
\end{aligned}
$$

Amplitude and phase variations are due to various causes, such as shifts in the reflecting layer or changes in the refraction index of the medium, which can modify the optical path of the radio link. So, the amplitudes and phases received can vary between shots: for the first shot, $A_{1}, \varphi_{1}$, for the second shot, $A_{2}, \varphi_{2}$. It will be assumed that the amplitude variations are negligible: $A_{1}=A_{2}=A$. Now the consequences of the variation on the phase can be calculated.

When the correlation process occurs, it converts the long sequences $I(t)$ and $Q(t)$ into short pulses, with a gain $K$, as stated in Equations (5.2) and (5.3). For the MBC method, from Equation (5.6):

$$
\begin{aligned}
& \text { МВСатр }=K\left(A_{1}+A_{2}\right)= \\
& =K\left(\sqrt{A^{2} \cos \varphi_{1}^{2}+A^{2} \sin \varphi_{1}^{2}}+\sqrt{A^{2} \cos \varphi_{2}^{2}+A^{2} \sin \varphi_{2}^{2}}\right)=2 K A,
\end{aligned}
$$

regardless of any difference between $\varphi_{1}$ and $\varphi_{2}$. For the QCC method, from Equation (5.7):

$$
\begin{gathered}
\text { QCCamp }=K A \sqrt{\begin{array}{l}
\cos ^{2} \varphi_{1}+\cos ^{2} \varphi_{2}+2 \cos \varphi_{1} \cos \varphi_{2}+ \\
+\sin ^{2} \varphi_{1}+\sin ^{2} \varphi_{2}+2 \sin \varphi_{1} \sin \varphi_{2}
\end{array}}= \\
=K A \sqrt{2} \cdot \sqrt{1+\cos \varphi_{1} \cos \varphi_{2}+\sin \varphi_{1} \sin \varphi_{2}}
\end{gathered}
$$

If $\varphi_{1}=\varphi_{2}$, the square root becomes $\sqrt{1+\cos ^{2} \varphi+\sin ^{2} \varphi}$ $=\sqrt{2}$, so Equation (B.1) becomes Equation (5.9). In all other cases, assuming $\varphi_{2}=\varphi_{1}+\Delta \varphi$, the variable term can be written as $\sqrt{1+\cos \Delta \varphi}<\sqrt{2}$. Consequently, QCCamp is always $\leq$ MBCamp. A Mathcad ${ }^{\mathcal{C}}$ simulation confirmed this result. Figure 9 shows the gain in the $\mathrm{dB}$ of the MBC method with respect to the QCC method for phase differences of up to $90^{\circ}$ (positive or negative).

The $3 \mathrm{~dB}$ gain that corresponds to the $90^{\circ}$ phase
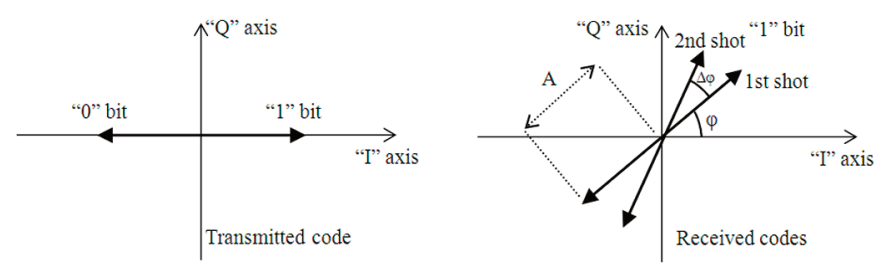

Figure 8. Complex representation of transmitted and received signals.

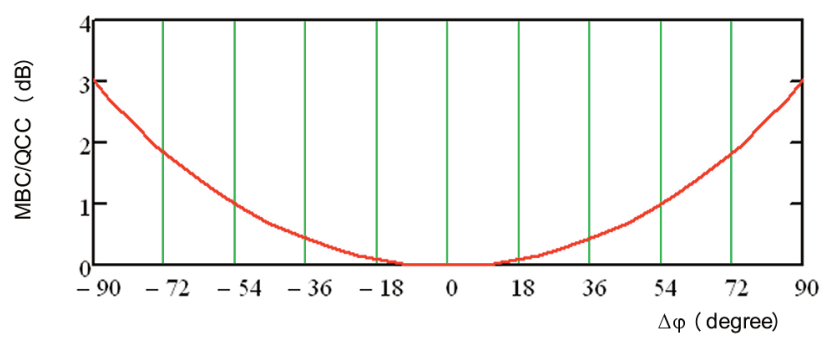

Figure 9. Gain (dB) of the MBC amplitude output with respect to QCC.

difference can also be deduced from Equation (B.1). Indeed, in this case, $\sqrt{1+\cos \varphi_{1} \cos \varphi_{2}+\sin \varphi_{1} \sin \varphi_{2}}=1$. It can be noted that a phase difference of $90^{\circ}$ or more is very unlikely to happen.

In all of the previous calculations, the signal received was meant to have zero mean value (in Figure 8 , the vectors are applied in the origin of the axes), i.e. the ADC in Figures 2 and 3 have zero bias outputs. All of the ADCs do actually show some bias, so they have to be put to zero (electronically) before starting any signal processing. Indeed, mathematical analysis of the signal with a bias would become much more difficult, and the signal outputs would risk being distorted.

\section{Appendix B}

Before calculating the noise power at the output of the two systems, it is useful to recall what happens when noise passes through the various types of the fundamental blocks that appear in the block diagrams. In all cases, when exiting a single block, it is assumed that the noise can vary its properties, that the mean value $(\eta)$ may change, and that if the power spectral density is constant over a band $B$, the variance $\sigma^{2}$ will be: $\sigma^{2}=B N^{2}$, where $N$ is the noise power spectral density. There now follows the output evaluations of the various blocks [Carlson 1986, Zingales 1992].

\section{Sum}

The output is the sum of the two inputs. If the input noises are not correlated, the output has the same characteristics, and the output power is the sum of the input powers:

$$
\sigma_{o}^{2}=\sigma_{1}^{2}+\sigma_{2}^{2} .
$$

If the inputs have the same power:

$$
\sigma_{o}^{2}=2 \sigma_{i}^{2}
$$

which happens when the result of the correlation of two codes 
are added into the memory (QCC case). In the MBC system, the input noise passes through the two branches of the quadrature demodulator; the two outputs can be considered not correlated, as in the QCC case, or even the same. In this second case the expected values are summed, giving:

$$
\sigma_{o}=2 \sigma_{i}
$$

In any case, the mean value is not affected by this block (if present at the inputs, they simply sum).

\section{Correlation}

The correlator is equivalent to a filter with a defined frequency response $C(\omega)$ over a limited bandwidth. This is a linear system, so zero mean noise at the input generates zero mean noise at the output. However, if $\eta_{i}$ is not zero, the output can be calculated simply (in general $C(0) \neq 0$ ) by:

$$
\eta_{o}=\eta_{i} \cdot C(0)
$$

If this kind of filter has a flat response over the same bandwidth of the input noise, the output has:

$$
\sigma_{o}^{2}=\sigma_{i}^{2}
$$

If the response is not flat, it is possible to define the noise equivalent bandwidth (NEB) as:

$$
N E B=\frac{1}{2 \pi} \int|C(\omega)|^{2} d \omega .
$$

In this way, the output noise power is given by:

$$
\sigma_{o}^{2}=N^{2} \cdot N E B=\sigma_{i}^{2} \cdot N E B / B .
$$

This last expression shows that if $N E B<B$, then the noise power decreases. We will assume that reasonably $N E B=B$; indeed, the noise entering the correlation filter has already passed through the IF filter.

\section{Square}

This operation makes the noise change its characteristics. Specifically, a non-zero mean value appears:

$$
\eta_{o}=\eta_{i}^{2}+\sigma_{i}^{2} .
$$

While the output variance is:

$$
\sigma_{o}^{2}=2 \sigma_{i}^{4}
$$

\section{Square root}

This operation is usually not analyzed in texts dealing with noise. The output variance can be inferred by calculating the inverse of the last result:

$$
\sigma_{o}^{2}=\sigma_{i} / 2
$$

For the mean value (assuming that the sign is reconstructed in some way):

$$
\eta_{o}=\sqrt{\eta_{i}}+\sqrt{\sigma_{i}} .
$$

Finally, we can predict the output power of the two systems, for the case of equal noise at the output of the two mixers. In the following schemes each line reports the output of the cascaded blocks of the two systems, assuming $\sigma^{2}$ be the variance at the input of each $I$ and $Q$ branch:

\section{MBC}

$\begin{array}{ll}\text { squaring: } & 2 \sigma^{4} \\ \text { adding the two branches output: } & 8 \sigma^{4} \\ \text { extracting the square root: } & 2 \sigma^{2} \\ \text { after correlation: } & 2 \sigma^{2} \\ \text { adding the results for two codes: } & 4 \sigma^{2}\end{array}$

\begin{tabular}{lr}
\hline \multicolumn{1}{c}{ QCC } \\
\hline after correlation: & $\sigma^{2}$ \\
adding the results for two codes: & $2 \sigma^{2}$ \\
squaring: & $8 \sigma^{4}$ \\
adding the two branches output: & $32 \sigma^{4}$ \\
extracting the square root: & $4 \sigma^{2}$ \\
\hline
\end{tabular}

So the outputs are the same. If the noises exiting the mixers are not meant to be correlated, the previous results are almost the same, except for the summation of the two branches that is seen, which makes the variance double instead of becoming four times the inputs. The result becomes

\begin{tabular}{|c|c|}
\hline \multicolumn{2}{|l|}{ MBC } \\
\hline squaring: & $\sigma^{2}$ \\
\hline adding the two branches output: & $2 \sigma^{2}$ \\
\hline extracting the square root: & $(\sqrt{2}+\sqrt[4]{2}) \sigma$ \\
\hline after correlation: & $C(0)(\sqrt{2}+\sqrt[4]{2}) \sigma$ \\
\hline adding the results for two codes: & $2 C(0)(\sqrt{2}+\sqrt[4]{2}) \sigma$ \\
\hline
\end{tabular}
$2 \sqrt{2} \sigma$, although it remains equal in the two systems.

\section{Mean values}

None of the calculations just performed considered the mean values, although similar calculations can be carried out to predict what happens to the means. In all cases, it is assumed that the input noise has a zero mean value $(\eta=0)$. For the two systems the mean noise values at the output of each block become: 


\section{QCC}

after correlation:

0

adding the results for two codes:

0

squaring:

$2 \sigma^{2}$

adding the two branches output:

$4 \sigma^{2}$

extracting the square root:

$$
2(\sqrt{2}+\sqrt[4]{2}) \sigma
$$

So, the two systems do not produce the same result. In general it is not possible to state the value $C(0)$, so the $\mathrm{MBC}$ system appears to be better than the QCC system if $C(0)<1$, or worse than the QCC system if $C(0)>1$.

\section{Appendix C}

Both the MBC and QCC algorithms produce time series of $n$ elements $x_{i}$ for which the power of the signal and the noise should be evaluated. For the simple case of a single echo, only a few points emerge from the noise, and amongst these, the absolute maximum value will correspond to the energy coming from the reflecting layer. For all of the series presented in this study, the variance of the series $\sigma^{2}$ is taken as the noise power evaluation. The SNR is then calculated in the following way:

$$
S N R=10 \log \frac{A^{2}}{\sigma^{2}}=10 \log \frac{(n-1) A^{2}}{\sum_{i}\left(x_{i}-\bar{x}\right)^{2}} .
$$

